\title{
A Plane Stress FEA Problem Which Students Can Solve Using an Inexpensive Calculator
}

\author{
Patrick J. Cronin \\ The Pennsylvania State University \\ New Kensington Campus
}

\begin{abstract}
This paper describes a stress analysis problem which can be used to introduce lower division engineering or engineering technology students to the finite element analysis (FEA) method. Step by step the student sets up the matrix equation which represents the system of simultaneous linear equations which is necessary to solve for the unknown displacements at each of the nodes. They then solve this system of equations using a numerical method which is efficient for large systems of simultaneous linear equations. Using these nodal displacements they calculate the normal and shear stresses at several different locations within the finite elements. These stresses are calculated by using the stress-displacement system of equations. This system of equations is also set up by the students. All of this they can do with an inexpensive scientific calculator.
\end{abstract}

Description of the Symbols Used.

\begin{tabular}{|c|c|}
\hline Symbol & Description \\
\hline $\mathrm{A}_{\mathrm{ij}}$ & elements of the matrix $[\mathrm{A}]$ \\
\hline $\mathrm{a}$ & width of the finite element \\
\hline b & height of the finite element \\
\hline$c_{i}$ & constants \\
\hline $\mathrm{D}_{\mathrm{ij}}$ & elements of the stress-displacement matrix \\
\hline $\mathrm{E}$ & modulus of elasticity \\
\hline $\mathrm{F}_{\mathrm{i}}$ & elements of the force matrix \\
\hline $\mathrm{k}_{\mathrm{ij}}$ & elements of the stiffness matrix \\
\hline $1_{\mathrm{ij}}$ & elements of lower triangular matrix [L] \\
\hline $\mathrm{t}$ & thickness of the finite element \\
\hline $\mathrm{u}_{\mathrm{i}}$ & elements of the displacement matrix \\
\hline $\mathrm{u}_{\mathrm{x}}$ & displacement in the $\mathrm{x}$-direction \\
\hline $\mathrm{u}_{\mathrm{y}}$ & displacement in the $y$-direction \\
\hline $\mathrm{v}_{\mathrm{i}}$ & elements of the matrix [v] \\
\hline $\mathrm{x}$ & coordinate along the horizontal axis \\
\hline $\mathrm{y}$ & coordinate along the vertical axis \\
\hline
\end{tabular}

Proceedings of the 2001 American Society for Engineering Education Annual Conference \& Exposition Copyright (C) 2001, American Society for Engineering Education 
Description of the Symbols Used ( continued )

\begin{tabular}{|c|c|}
\hline Symbol & $\frac{\text { Description }}{\text { ( Greek letters ) }}$ \\
\hline$\beta$ & aspect ratio (i.e. b/a) \\
\hline$\eta$ & dimensionless coordinate (vertical axis ) \\
\hline$v$ & Poisson's ratio \\
\hline$\xi$ & dimensionless coordinate ( horizontal axis ) \\
\hline$\sigma_{\mathrm{xx}}$ & normal stress in the $\mathrm{x}$-direction \\
\hline$\sigma_{y y}$ & normal stress in the $y$-direction \\
\hline$\sigma_{\mathrm{xy}}$ & shear stress in the xy-plane \\
\hline
\end{tabular}

\section{Introduction}

Finite element analysis software is widely used to calculate the normal and shear stresses in mechanical parts and structures of many different shapes and sizes. The types of applied loadings can be concentrated forces or distributed loadings or combinations of both. A student being introduced to the calculation of stresses can benefit from being exposed to the terminology and progression of calculations used to calculate stresses by the finite element method. Some of the applicable finite element terms are: nodes, degrees of freedom, element coordinate axes, global coordinate system, stiffness matrices, forward reduction, and backward substitution. This paper does not imply that hand calculations of stress are preferable to the use of finite element software. The benefit of this exercise is some insight into finite element calculation procedures.

An element which is quite useful and yet not too complicated is the rectangular element with corner nodes. ${ }^{1,2}$ Details of this element can also be found. ${ }^{3,4}$ This element is shown in Figure 1. The local coordinate axes ( i.e. $\mathrm{x}$ and $\mathrm{y}$ ) are shown, as well as the nondimensional coordinates

$$
\xi=\frac{x}{a} \quad \text { and } \quad \eta=\frac{y}{b}
$$

The displacement functions for the element in terms of the nondimensional coordinates are

$$
\begin{aligned}
& \mathrm{u}_{\mathrm{x}}=\mathrm{c}_{1} \xi+\mathrm{c}_{2} \xi \eta+c_{3} \eta+c_{4} \\
& \mathrm{u}_{\mathrm{y}}=\mathrm{c}_{5} \xi+c_{6} \xi \eta+c_{7} \eta+c_{8}
\end{aligned}
$$

where $\mathrm{c}_{1}$ through $\mathrm{c}_{8}$ are functions of the nodal displacements $\mathrm{u}_{1}$ through $\mathrm{u}_{8}$. 


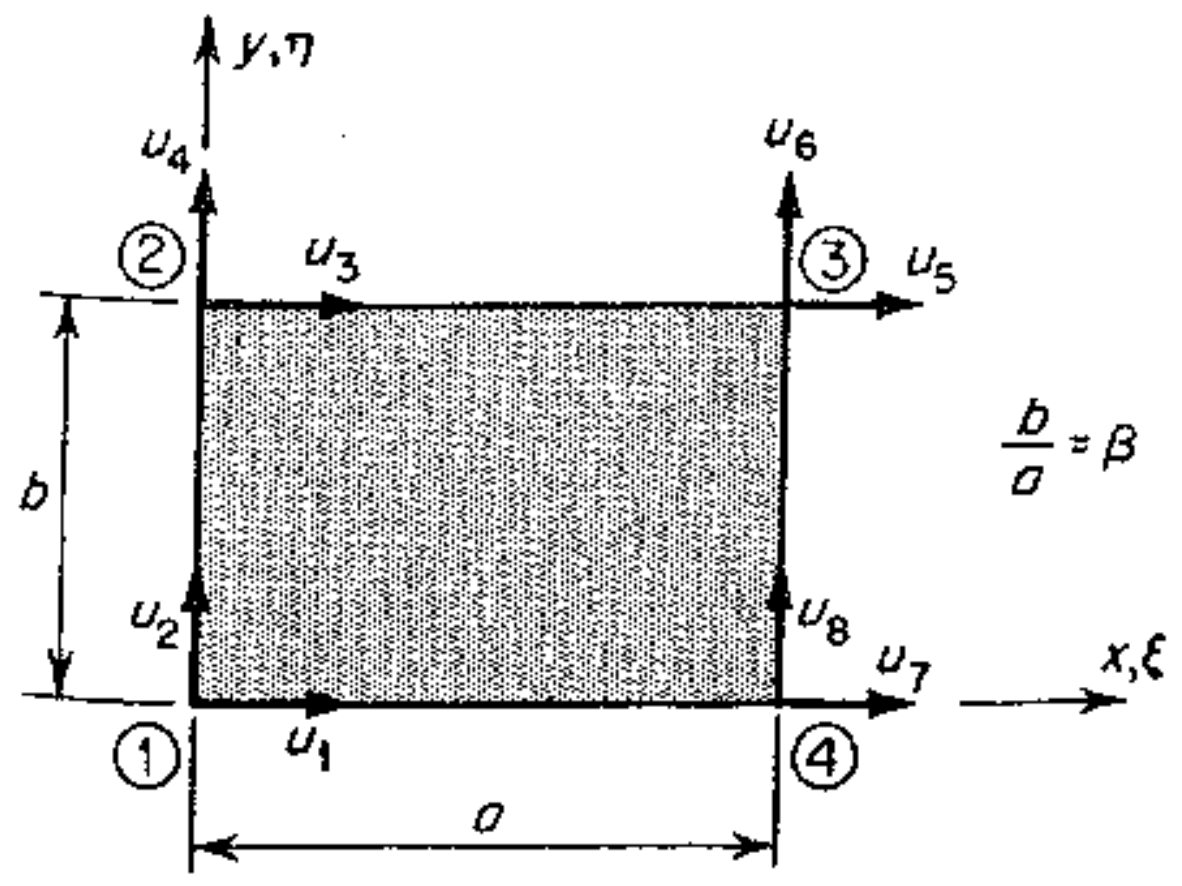

Figure 1. Rectangular plane-stress finite element. [1]

It will be shown later in this paper that these displacement functions allow for linear variation of the stresses in the horizontal and vertical directions.

\section{Problem Description.}

A square plate to be analyzed for stresses is shown in Figure 2. Some plate displacements are constrained as shown. The applied forces are as shown there. The objective is to calculate the approximate $\sigma_{\mathrm{xx}}, \sigma_{\mathrm{yy}}$, and $\sigma_{\mathrm{xy}}$ stresses at the 20 points which are at the locations shown in Figure 2. The plate is 20 inches by 20 inches square and is made of steel. The material properties are $\mathrm{E}=30.0 \mathrm{E} 6 \mathrm{lb} / \mathrm{in}^{2}$ and $v=0.30$. The thickness is 0.3640 inches. The problem can be solved by using one-fourth of the plate (see Figure 3 ) because of the symmetry of the loading and boundary conditions about the horizontal and vertical center lines. The stresses at the 5 points in quadrants I, III, and IV of the plate shown in Figure 2 can be deduced from the stresses at the 5 points in quadrant II ( upper right quadrant). 


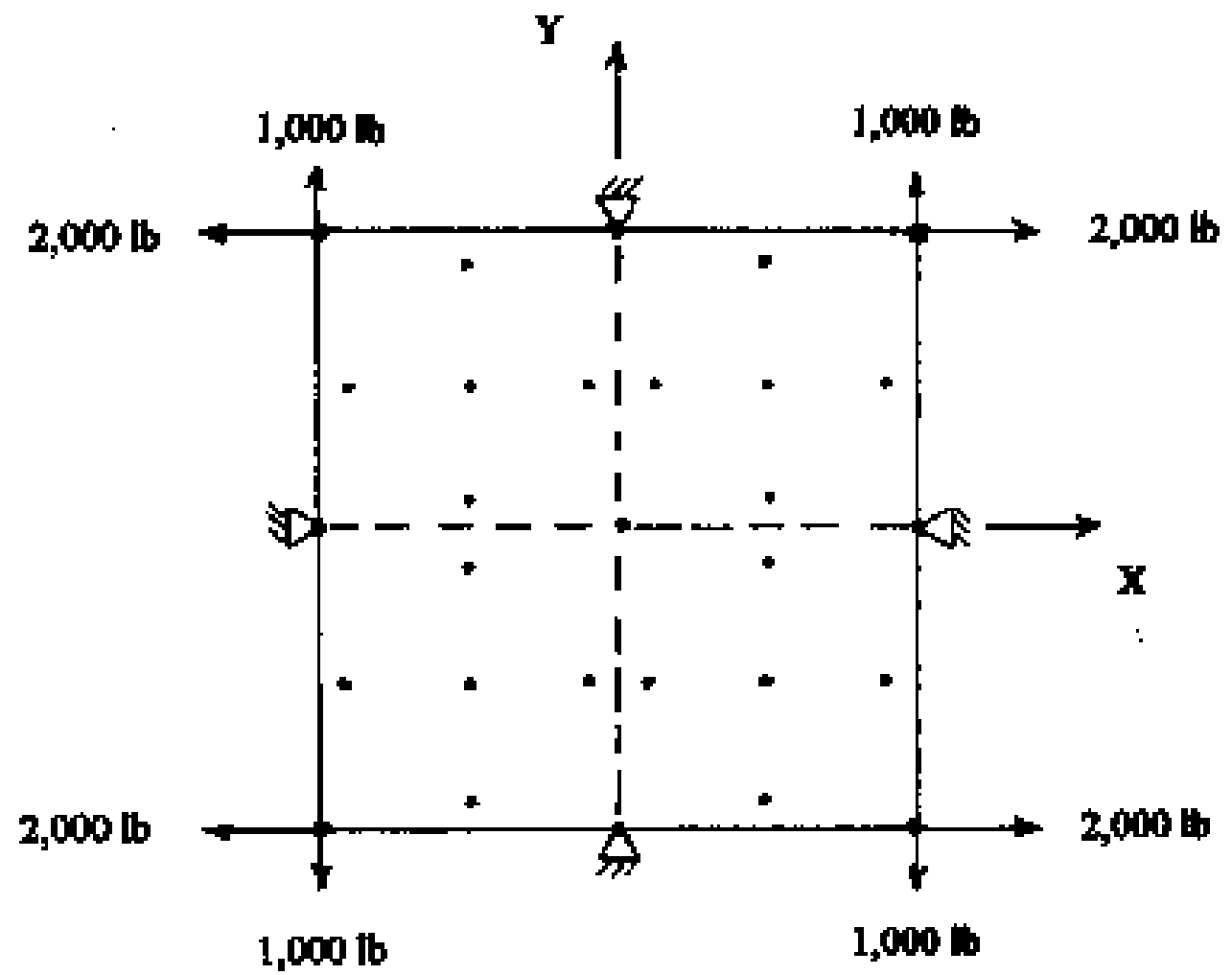

Figure 2. Plate ( 20 in. $x 20$ in. ) to be analyzed for stresses.

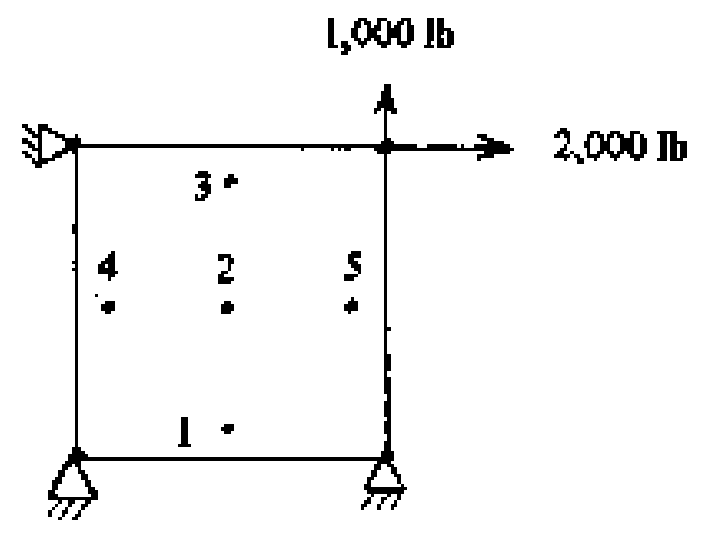

Figure 3. The quadrant, of the original plate, which will be analyzed. 


\section{Problem Solution.}

The first step is to calculate the displacements in the $\mathrm{x}$ and $\mathrm{y}$ directions at the upper right corner. This is done by using the matrix equation

$$
[K]\{u\}=\{F\}
$$

which is based on the FEA method. In this equation

$$
\begin{aligned}
& {[K]=\text { stiffness matrix }} \\
& \{u\}=\text { column matrix of displacements } \\
& \{F\}=\text { column matrix of forces }
\end{aligned}
$$

The element to be used is shown in Figure 1 which is taken from Reference 1 . All of the displacements are zero except $\mathrm{u}_{5}$ and $\mathrm{u}_{6}$. Based upon equation 5.172 of reference 1

$$
[K]=\frac{E t}{12\left(1-v^{2}\right)}\left[\begin{array}{ll}
A_{11} & A_{12} \\
A_{21} & A_{22}
\end{array}\right]
$$

where

$$
\begin{aligned}
& \mathrm{A}_{11}=4 \beta+2(1-v) / \beta=5.4 \overline{0} \\
& \mathrm{~A}_{21}=\mathrm{A}_{12}=\frac{3}{2}(1+v)=1.95 \overline{0} \\
& \mathrm{~A}_{22}=4 / \beta+2(1-v) \beta=5.4 \overline{0}
\end{aligned}
$$

The $\beta$ value is 1.0 . It is the aspect ratio of the rectangular element which is the height of the rectangle divided by the width of the rectangle.

$$
\frac{E t}{12\left(1-v^{2}\right)} \text { is equal to } 1.0 \overline{0} \mathrm{E} 6 .
$$

Therefore, the matrix equation to be used to solve for the corner displacements is

$$
\left[\begin{array}{cc}
5.4 \overline{0} & 1.95 \overline{0} \\
1.95 \overline{0} & 5.4 \overline{0}
\end{array}\right]\left[\begin{array}{l}
u_{5} \\
u_{6}
\end{array}\right]=\left[\begin{array}{l}
.002 \overline{0} \\
.001 \overline{0}
\end{array}\right]
$$

Both sides of the equation have been divided by $1.0 \overline{0} \mathrm{E} 6$. 
This modest matrix equation representing a pair of simultaneous equations with 2 unknowns will be solved by using Choleski's method. This method is demonstrated because it is more practical than Cramer's rule, for example, for large systems of simultaneous equations. Using Choleski's method to solve the above matrix equation, we have

$$
\begin{aligned}
& k_{11}=k_{22}=5.4 \overline{0} \text { and } k_{21}=k_{12}=1.95 \overline{0} \\
& l_{11}=k_{11}^{1 / 2}=(5.4 \overline{0})^{0.5}=2.3238 \\
& l_{21}=\frac{k_{21}}{l_{11}}=\frac{1.95 \overline{0}}{2.3238}=0.83914 \\
& l_{22}=\left(k_{22}-l_{21}^{2}\right)^{0.5}=2.1670
\end{aligned}
$$

where $l_{11}, l_{21}$, and $l_{22}$ are elements of a lower triangular matrix designated as $\mathrm{L}$. The forward reduction process of Choleski's method is

$$
\mathrm{Lv}=\mathrm{F}
$$

so

$$
\left[\begin{array}{cc}
2.3238 & 0 \\
0.83914 & 2.1670
\end{array}\right]\left[\begin{array}{l}
\mathrm{v}_{1} \\
\mathrm{v}_{2}
\end{array}\right]=\left[\begin{array}{l}
.002 \\
.001
\end{array}\right]
$$

The first equation easily yields

$$
\mathrm{v}_{1}=8.6066 \mathrm{E}-4
$$

and the second equation gives

$$
\mathrm{v}_{2}=1.2819 \mathrm{E}-4
$$

The backward substitution of Choleski's method is

$$
\mathrm{L}^{\mathrm{T}} \mathrm{u}=\mathrm{v}
$$

giving

$$
\left[\begin{array}{cc}
2.3238 & 0.83914 \\
0 & 2.1670
\end{array}\right]\left[\begin{array}{l}
u_{1} \\
u_{2}
\end{array}\right]=\left[\begin{array}{l}
8.6066 E-4 \\
1.2819 E-4
\end{array}\right]
$$

The second of these equations easily gives

$$
\mathrm{u}_{2}=5.9156 \mathrm{E}-5
$$

Proceedings of the 2001 American Society for Engineering Education Annual Conference \& Exposition 
and the first of these equations yields

$$
\mathrm{u}_{1}=3.4901 \mathrm{E}-4
$$

The student should be required to check these two values by substituting them back into the original two equations. This will ensure that the ensuing stress calculations will not have to be repeated later due to the use of erroneous displacement values in the first stress calculation. Based upon equation 5.171 of reference 1

$$
\{\sigma\}=\frac{E}{1-v^{2}}[D]\{u\}
$$

where

$$
\begin{aligned}
& \{\sigma\}=\text { column matrix of stresses } \\
& \frac{E}{1-v^{2}}[D]=\text { stress }- \text { displacement matrix } \\
& \{u\}=\text { column matrix of displacements }
\end{aligned}
$$

In this matrix equation
$\mathrm{D}_{11}=\frac{\eta}{a}$
$\mathrm{D}_{12}=\frac{v \xi}{b}$
$\mathrm{D}_{21}=\frac{v \eta}{a}$
$\mathrm{D}_{22}=\frac{\xi}{b}$
$\mathrm{D}_{31}=\frac{(1-v) \xi}{2 b}$
$\mathrm{D}_{32}=\frac{(1-v) \eta}{2 a}$

this gives

$$
\begin{aligned}
& \sigma_{x x}=\frac{E}{1-v^{2}}\left[\left[\frac{\eta}{a}\right] u_{5}+\left[\frac{v \xi}{b}\right] u_{6}\right] \\
& \sigma_{\mathrm{yy}}=\frac{E}{1-v^{2}}\left[\left[\frac{v \eta}{a}\right] u_{5}+\left[\frac{\xi}{b}\right] u_{6}\right] \\
& \sigma_{\mathrm{xy}}=\frac{E}{1-v^{2}}\left[\left[\frac{(1-v) \xi}{2 b}\right] u_{5}+\left[\frac{(1-v) \eta}{2 a}\right] u_{6}\right]
\end{aligned}
$$

Substituting values for everything except $\xi$ and $\eta$

$$
\begin{aligned}
& \sigma_{\mathrm{xx}}=58.55 \xi+1151 \eta \\
& \sigma_{\mathrm{yy}}=195.2 \xi+345.2 \eta \\
& \sigma_{\mathrm{xy}}=402.7 \xi+68.31 \eta
\end{aligned}
$$

Proceedings of the 2001 American Society for Engineering Education Annual Conference \& Exposition Copyright $\odot$ 2001, American Society for Engineering Education 


\section{Stress Distribution}

The linear variation of $\sigma_{\mathrm{xx}}$ in the vertical direction can be demonstrated by calculating $\sigma_{\mathrm{xx}}$ at points 1,2, and 3 on the vertical straight line ( see Figure 3 ). The element coordinates ( i.e. ( $\xi$, $\eta)$ ) would be $\quad(0.5,0.1),(0.5,0.5)$, and $(0.5,0.9)$.

.These calculations are as follows

$$
\begin{aligned}
& \sigma_{\mathrm{xx}}(0.5,0.1)=(58.55)(0.5)+(1151)(0.1)=144 \mathrm{psi} \\
& \sigma_{\mathrm{xx}}(0.5,0.5)=(58.55)(0.5)+(1151)(0.5)=605 \mathrm{psi} \\
& \sigma_{\mathrm{xx}}(0.5,0.9)=(58.55)(0.5)+(1151)(0.9)=1065 \mathrm{psi}
\end{aligned}
$$

This corresponds to the straight line equation

$$
\sigma_{\mathrm{xx}}=1151 \eta+29.28
$$

Similarly, the straight line equation for $\sigma_{\mathrm{yy}}$ along $\xi=0.5$ becomes

$$
\sigma_{\mathrm{yy}}=195.2 \eta+172.6
$$

and the straight line equation for $\sigma_{\mathrm{xy}}$ along $\xi=0.5$ becomes

$$
\sigma_{\mathrm{xy}}=402.7 \eta+34.2
$$

The linear variation along the $\xi=0.5$ vertical line is displayed in Table 1 .

$\begin{array}{lccc}\eta & \underline{\sigma}_{\underline{x x}}(\mathbf{p s i}) & \underline{\sigma}_{y y}(\mathrm{psi}) & \underline{\sigma}_{\underline{x y}}(\mathrm{psi}) \\ 0.1 & 144 & 132 & 208 \\ 0.5 & 605 & 270 & 236 \\ 0.9 & 1,065 & 408 & 263\end{array}$

Table 1. Linear Variations of $\sigma_{\mathrm{xx}}, \sigma_{\mathrm{yy}}$ and $\sigma_{\mathrm{xy}}$ along vertical line $\xi=0.5$

The linear variation along the horizontal line formed by points 4,2 , and 5 at $\eta=0.5$ ( see Figure 3 ) can be represented by the 3 straight line equations

$$
\begin{aligned}
& \sigma_{\mathrm{xx}}=(58.55) \xi+575.5 \\
& \sigma_{\mathrm{yy}}=(195.2) \xi+172.6 \\
& \sigma_{\mathrm{xy}}=(402.7) \xi+34.2
\end{aligned}
$$

The linear variation along the $\eta=0.5$ horizontal line is displayed Table 2 . 


$\begin{array}{cccc}\xi & \underline{\sigma}_{\underline{x x}}(p s i) & \underline{\sigma}_{y \underline{y}}(p s i) & \underline{\sigma}_{\underline{x y}}(p s i) \\ 0.1 & 581 & 192 & 74 \\ 0.5 & 605 & 270 & 236 \\ 0.9 & 628 & 348 & 397\end{array}$

Table 2. Linear Variations of $\sigma_{x x}, \sigma_{y y}$, and $\sigma_{x y}$ along horizontal line $\eta=0.5$.

\section{Summary}

The calculations of some approximate normal and shear stresses in a thin square metal plate has been demonstrated using a very coarse finite element grid. This calculation makes use of a rectangular finite element with two displacement degrees of freedom at each corner node. This element makes possible the calculation of stresses which vary linearly in both the horizontal and vertical directions. Symmetry of the applied loads and of the boundary conditions is made use of to reduce the size of the model by $75 \%$. The metal plate approximate stresses are calculated by the students using the same types of matrix equations which would be solved by a computer using much larger systems of equations based upon several hundred or more rectangular elements to represent the plate. One benefit of the exercise is the acquiring of some familiarity with the FEA symbols, terminology, and matrix equations. Another benefit is an understanding of the mechanics of a solution algorithm for large systems of simultaneous linear equations.

References

1. Przemieniecki, J.S.,Theory of Matrix Structural Analysis, Dover Publications, Inc., New York, 1985.

2. Argyrus, Energy Theorems and Structural Analysis, Butterworth Scientific Publications, London, 1960.

3. Przemieniecki, J.S., and L. Berke: "Digital Computer Program for the Analysis of Aerospace Structures by the Matrix Displacement Method", Flight Dynamics Lab. Report FDL-TDR 64-18, 1964.

4. Turner, M.J., R.W. Clough, H.C. Martin, and L.J. Topp: "Stiffness and Deflection Analysis of Complex Structures", Journal of Aeronautical Sciences, 23 : 805-823, 854 (1956).

PATRICK J. CRONIN

Patrick J. Cronin is currently an Assistant Professor of Engineering at The Pennsylvania State University New Kensington Campus. He holds a B.S. in Engineering Science and an M..S. degree in Civil Engineering, both from the State University of New York at Buffalo. He previously worked as an engineer performing stress and vibration analysis of various types of mechanical equipment. 